\title{
A Comparison Between the Korean Digits-in-Noise Test and the Korean Speech Perception-in-Noise Test in Normal-Hearing and Hearing-Impaired Listeners
}

\author{
Subin Kim ${ }^{1}$, Sungwha You ${ }^{2,3}$, Myoung Eun Sohn ${ }^{1}$, Woojae Han ${ }^{2,3}$, Jae-Hyun Seo ${ }^{1}$, and Yonghee $\mathrm{Oh}^{4}$ \\ ${ }^{1}$ Department of Otolaryngology-Head and Neck Surgery, College of Medicine, The Catholic University of Korea, Seoul, Korea \\ ${ }^{2}$ Laboratory of Hearing and Technology, Research Institute of Audiology and Speech Pathology, College of Natural Sciences, Hallym \\ University, Chuncheon, Korea \\ ${ }^{3}$ Division of Speech Pathology and Audiology, College of Natural Sciences, Hallym University, Chuncheon, Korea \\ ${ }^{4}$ Department of Speech, Language, and Hearing Sciences, University of Florida, Gainesville, FL, USA
}

\author{
Received February 1, 2021 \\ Revised June 11, 2021 \\ Accepted July 21, 2021
}

Background and Objectives: The purpose of the present study was to validate the performance and diagnostic efficacy of the Korean digits-in-noise (K-DIN) test in comparison to the Korean speech perception-in-noise (K-SPIN) test, which is the representative speech-in-noise test in clinical practice. Subjects and Methods: Twenty-seven subjects (15 normal-hearing and 12 hearing-impaired listeners) participated. The recorded Korean 0-9 digits were used to form quasirandom digit triplets; 50 target digit triplets were presented at the most comfortable level of each subject while presenting speech-shaped background noise at various levels of signal-to-noise ratios $(-12.5,-10,-5$, or $+5 \mathrm{~dB})$. Subjects were then instructed to listen to both target and noise masker unilaterally and bilaterally through a headphone. K-SPIN test was also conducted using the same procedure as the K-DIN. After calculating their percent correct responses, K-DIN and K-SPIN results were compared using a Pearson-correlation test. Results: Results showed a statistically significant correlation between K-DIN and K-SPIN in all hearing conditions (left: $r=0.814, p<0.001$; right: $r=0.788, p<0.001$; bilateral: $r=0.727$, $p<0.001)$. Moreover, the K-DIN test achieved better testing efficacy, shorter average listening time (5 min vs. 30 min), and easier performance of task according to participants' qualitative reports than the K-SPIN test. Conclusions: In this study, the Korean version of digit triplet test was validated in both normal-hearing and hearing-impaired listeners. The findings suggest that the K-DIN test can be used as a simple and time-efficient hearing-in-noise test in audiology clinics in Korea.

J Audiol Otol 2021;25(4):171-177

Keywords: Digits-in-noise test; Sensorineural hearing loss; Speech perception.

\section{Introduction}

In an aging society, the number of patients with sensorineural hearing loss is increasing. Difficulty of hearing in noise is one of the first signs of sensory neural hearing loss [1]. Therefore, an efficient speech perception-in-noise (SPIN) test is essential for the early detection of hearing disorders. A self-

This is an Open Access article distributed under the terms of the Creative Commons Attribution Non-Commercial License (https://creativecommons.org/licenses/by-nc/4.0/) which permits unrestricted non-commercial use, distribution, and reproduction in any medium, provided the original work is properly cited. screening-test that can be easily administered allows to diagnose hearing loss early and to initiate interventions such as selection of hearing aids and auditory rehabilitation.

Various types of hearing-in-noise tests have been developed using several speech materials. Among them, sentencein-noise tests are well suited to measure speech communication in everyday listening conditions as the sentences involve high-level linguistic, contextual, and cognitive factors [2]. This includes the hearing-in-noise test [3], the quick speech-innoise test [4], and the matrix sentence test [5]. For example in Korea, the Korean speech perception-in-noise (K-SPIN) test 
was developed to consider the Korean characteristics [6] based on the English version of the SPIN [7] at a fixed signalto-noise ratio (SNR). The Korean version of the hearing-innoise test (K-HINT) is developed jointly with the House Ear Institute of the United States using an adaptive SNR method [8]. The matrix sentence test, which has a minimal training effect and is repeatable, has a Korean version as well [9].

On the other hand, a digit is a simple speech material than a sentence composed of words universally. Digits are a highly familiar speech material so they are relatively immune to learning effects, linguistic ability factors, and personal factors [10]. In detail, they are repeatable due to a small learning effect compared to sentences and/or words [11]. When the audience answers using a digital keyboard, there is no need for the examiner to score the response which makes automatic screening possible [12]. Moreover, a digit is relatively independent from language which makes multilingual comparison across languages available [13].

To improve detection of hearing loss, the digit-in-noise (DIN) test was originally developed as a self-screening speech-innoise test over the telephone in Dutch [4]. This speech-innoise test measure the SNR where a listener recognizes 50\% of the digit-triplets correctly. The earliest version of the DIN test was successful as a screening test, allowing over 65,000 calls during first 4 months before it was later implemented as an online test. Successful test performance led to the development of DIN tests in different languages. In the last decade, many studies have shown the effectiveness of the DIN test as a screening test in language like British English [14], American English [15], German [16], French [17], Mandarin [18], Polish [19], Russian [20], and Spanish [21].

Recently, the Korean version of the digit triplet test has been developed and optimized for mobile testing devices for normal hearing $(\mathrm{NH})$ adult listeners [22]. In that study, Korean monosyllabic digits $0-9$ were used, and optimization showed comparable results to the different language versions of the digit triplet test. There was no statistical difference among four different mobile devices in terms of average thresholds of the speech recognition [23]. However, the Korean version of the digit triplet test has not yet been compared with the current Korean sentence tests nor validated for hearing-impaired listeners, consequently not being verified for the clinical usage. Therefore, the purpose of our study is to validate the performance and diagnostic efficacy of the Korean version of the digits-in-noise (K-DIN) test as a speech-in-noise screening test.

\section{Subjects and Methods}

\section{Subjects}

These studies were conducted according to the guidelines for the protection of human subjects as set forth by the Institutional Review Board (IRB) of the School of Medicine, Catholic University of Korea, and the methods employed were approved by that IRB (approval No. HC18FESI0053). Informed consent was submitted by all subjects when they were enrolled. Twenty-seven Korean speakers (12 females and 15 males; mean age: $41 \pm 17$ years old) participated in the study. Fifteen subjects were NH listeners and twelve subjects were hearing-aid (HA) users. $\mathrm{NH}$ was defined as air conduction puretone thresholds $\leq 25 \mathrm{~dB}$ hearing level (HL) averaged across $0.5,1,2$, and $4 \mathrm{kHz}$ (weighted four-frequency average; W4FA). Mean pure-tone averages (PTAs) were $4 \pm 5$ and $4 \pm 4 \mathrm{~dB} \mathrm{HL}$ for left and right ears, respectively. All HA users had mild-tosevere hearing losses in both ears and relatively symmetric hearing losses between ears (mean PTAs: $43 \pm 16$ and $41 \pm 20$ dB HL), except for subjects HA1, HA11, and HA12. None of participants had profound hearing loss. All HA users had at least 1 year of experience with their HAs. The detailed HA users' demographic information including age, sex, PTA, most comfortable level (MCL), and Korean Mini-Mental Status Examination (K-MMSE) are shown in Table 1. Tympanometry was also conducted for all subjects to verify normal middle ear function. All subjects were screened for normal cognitive function using the 10-minute K-MMSE with a minimum score of 27 out of 30 required to qualify [24].

\section{Stimuli and procedures}

Two speech-in-nose tests were conducted in this study: the K-DIN test and the K-SPIN test. The results for all experiments were averaged with three separate runs for both K-DIN and K-SPIN in various experimental conditions: 1) three hearing conditions (left-only, right-only, and both ears), 2) various noise level conditions ( $\mathrm{NH}$ subjects: $-12.5,-10,-5$, and $+5 \mathrm{~dB}$ SNRs; HA subjects: -5 and $+5 \mathrm{~dB}$ SNRs). Note that two negative SNRs ( -12.5 and $-10 \mathrm{~dB}$ ) were only tested in NH subjects, because the tasks in those SNR conditions are too difficult for HA subjects, i.e., floor effect.

For the K-DIN, fifty unique digit triplets consisting of $0-9$ monosyllabic digits were created without repeating digits (e.g., "9-5-3") and mixed with speech-shaped background noise. The digits $(0-9)$ were recorded by a female news anchor working in Korea's broadcasting company using standard Korean pronunciation. The recorded digits were randomly selected to form digit triplets, thus no co-articulatory and prosodic cues were provided between digit triplets. The generated quasir- 
Table 1. Demographic information of the listeners with $\mathrm{NH}$ (averaged) and the listeners with HA (individual)

\begin{tabular}{|c|c|c|c|c|c|c|c|}
\hline \multirow{2}{*}{ Subject ID } & \multirow{2}{*}{ Age $(y r)$} & \multirow{2}{*}{ Sex } & \multicolumn{2}{|c|}{ PTA (W4FA, dB HL) } & \multicolumn{2}{|c|}{$M C L(d B S P L)$} & \multirow{2}{*}{ K-MMSE } \\
\hline & & & Left & Right & Left & Right & \\
\hline $\mathrm{NH}(\mathrm{n}=15)$ & $31 \pm 12$ & $F: M=7: 8$ & $4 \pm 5$ & $4 \pm 4$ & $63 \pm 4$ & $63 \pm 4$ & $30 \pm 1$ \\
\hline \multicolumn{8}{|l|}{$\mathrm{HA}(\mathrm{n}=12)$} \\
\hline $\mathrm{HAl}$ & 32 & $\mathrm{~F}$ & 22 & 49 & 80 & 85 & 30 \\
\hline HA2 & 31 & M & 18 & 24 & 60 & 60 & 28 \\
\hline HA3 & 56 & M & 44 & 31 & 75 & 60 & 29 \\
\hline HA4 & 61 & M & 56 & 44 & 90 & 80 & 30 \\
\hline HA5 & 68 & $\mathrm{~F}$ & 44 & 35 & 80 & 65 & 30 \\
\hline HAG & 73 & M & 58 & 51 & 90 & 90 & 30 \\
\hline HA7 & 56 & $\mathrm{~F}$ & 71 & 85 & 110 & 125 & 30 \\
\hline HA8 & 64 & $\mathrm{~F}$ & 59 & 50 & 90 & 90 & 30 \\
\hline HA9 & 43 & M & 30 & 19 & 70 & 60 & 30 \\
\hline $\mathrm{HA} 10$ & 53 & M & 44 & 32 & 75 & 70 & 30 \\
\hline HA 11 & 59 & M & 28 & 62 & 65 & 100 & 30 \\
\hline $\mathrm{HA} 12$ & 52 & $\mathrm{~F}$ & 42 & 4 & 80 & 50 & 30 \\
\hline Average & $54 \pm 13$ & $F: M=5: 7$ & $43 \pm 16$ & $41 \pm 20$ & $80 \pm 13$ & $78 \pm 20$ & $30 \pm 1$ \\
\hline
\end{tabular}

Data are presented as mean \pm standard deviation. Note that only group-averaged data shown for $\mathrm{NH}$ subjects. $\mathrm{NH}$, normal hearing; HA, hearing-aid; W4FA, weighted four-frequency average; PTA, pure-tone average; MCL, most comfortable level; HL, hearing loss; SPL, sound pressure level; K-MMSE, Korean Mini-Mental Status Examination

andom-50 digit triplets were then presented as a target at the most comfortable levels with speech-shaped background noise masker at various SNRs of $-12.5,-10,-5$, or $+5 \mathrm{~dB}$.

Prior to the K-DIN, digit loudness measurements were conducted in each ear separately using the following method of adjustment. First, digits were initialized to $65 \mathrm{~dB}$ sound pressure level (SPL) corresponding to a 6 or "most comfortable" on a visual loudness scale from 0 (no sound) to 10 (too loud). Loudness was then adjusted based on subject feedback. The averaged comfortable sound levels were $63 \pm 4 / 63 \pm 4 \mathrm{~dB}$ SPL (left/right ear) for NH listeners and $80 \pm 13 / 78 \pm 20 \mathrm{~dB}$ SPL (left/right ear) for HA users. MCLs were provided in detail in Table 1. In addition, the embedded background noise has the same spectrum as the long-term averaged digit spectrum. This digit shaped noise was generated by Adobe Audition (Adobe, San Jose, CA, USA).

For the K-SPIN, forty sentences were randomly selected from $240 \mathrm{~K}$-SPIN sentences without repeating and mixed with speech-shaped background noise. All K-SPIN sentences were recorded by the same female talker as used in the KDIN. Similar to the K-DIN, the background noise was generated with the same spectrum as the long-term averaged $\mathrm{K}$ SPIN sentence spectrum, the MCLs were estimated by the loudness measurement, and the target sentences were presented in various levels of the noise masker $(\mathrm{NH}:-12.5,-10,-5$, and $+5 \mathrm{~dB}$ SNR; HA: -5 and $+5 \mathrm{~dB}$ SNR).

Data collection was conducted in a single-wall, sound attenuated booth, All stimuli were generated at a sampling rate of $44.1 \mathrm{kHz}$ with Matlab (The MathWorks, Inc., Natick, MA, USA) on a Surface Pro computer (Microsoft, Redmond, WA, USA), processed through Babyface Pro external sound card (RME Audio, Haimhausen, Germany), and presented over Sennheiser HD 280 Pro headphones (Sennheiser Electronics GmbH \& Co. KG, Wedemark-Wennebostel, Germany). Each headphone's frequency response was equalized using calibration measurements obtained by a sound level meter with a 1-inch microphone in an artificial ear (Brüel \& Kjær, Nærum, Denmark). It should be noted that all signal levels for HA subjects were adjusted by the National Acoustics Laboratories prescription for non-linear formula (NAL-NL2) [23] in order to compensate for their hearing loss across frequencies.

For both K-DIN and K-SPIN, subjects were instructed to type the target speech through a Surface Pro touchscreen (Microsoft) as they heard it. The percentage of subject's correct responses were calculated based on the scoring rules: the correct responses are when all three digits and all three words were recognized for the K-DIN and K-SPIN, respectively. Both K-DIN and K-SPIN were performed in each monaural (i.e., left ear and right ear) and bilateral hearing conditions with all participants at each SNR level. Since $15 \mathrm{NH}$ subjects were tested at four fixed SNR levels $(-12.5,-10,-5$, and $+5 \mathrm{~dB})$ and $12 \mathrm{HA}$ subjects were tested at two fixed SNR levels ( -5 and $+5 \mathrm{~dB}$ ), there are 8 possible comparisons that can be observed between each test. All statistical analyses were conducted in SPSS Statistics 25.0 (IBM Corp., Armonk, NY, USA). In particular, a two-tailed Pearson correlation analysis (SPSS) 
was used to measure strength and direction of a linear relationship between the K-DIN and K-SPIN for each hearing condition (i.e., two monaural and binaural hearing).

\section{Results}

Fig. 1 shows the averaged accuracy scores ( \pm 1 standard deviation around the mean) of the K-DIN (upper panels) and the K-SPIN (lower panels) for NH listener (left panels) and HA users (right panels). For NH listeners, mean accuracy scores in the K-DIN were about 50\% (range 28\%-78\%) at the lowest SNR $(-12.5 \mathrm{~dB})$ condition and improved up to nearly $100 \%$ (range $88 \%-100 \%$ ) at the $-5-\mathrm{dB}$ SNR condition, regardless of hearing condition (i.e., monaural vs. binaural). Similar improvement trends were also observed in the K-SPIN. On the contrary, HA users showed greater variability of the accuracy scores at the $-5-\mathrm{dB}$ SNR condition (K-DIN: range 28\%-100
$\%$; K-SPIN: range $0 \%-100 \%$ ) and even at the +5 -dB SNR condition (K-DIN: range 52\%-100\%; K-SPIN: range 20\%$100 \%)$.

A three-way ANOVA was performed with accuracy score (percent correct, \%) as the dependent variable, and hearing condition (monaural and binaural), test type (K-DIN and KSPIN), and listening conditions (SNR) as independent variables. Note that listener groups ( $\mathrm{NH}$ and HA) were not compared because their SNR conditions were different (NH: -12.5 , $-10,-5$, and $+5 \mathrm{~dB}$ SNR; HA: -5 and $+5 \mathrm{~dB}$ SNR). The results showed a main effect of level differences between target and noise maskers (i.e., SNRs) on accuracy score $(p<0.001)$, and a marginal significant effect of hearing condition $(p=0.033)$. No significant effect of test type (K-DIN and K-SPIN) and interactions between all independent variables was observed ( $p>$ 0.145 for all cases).

The main goal of this study was to determine whether the K-
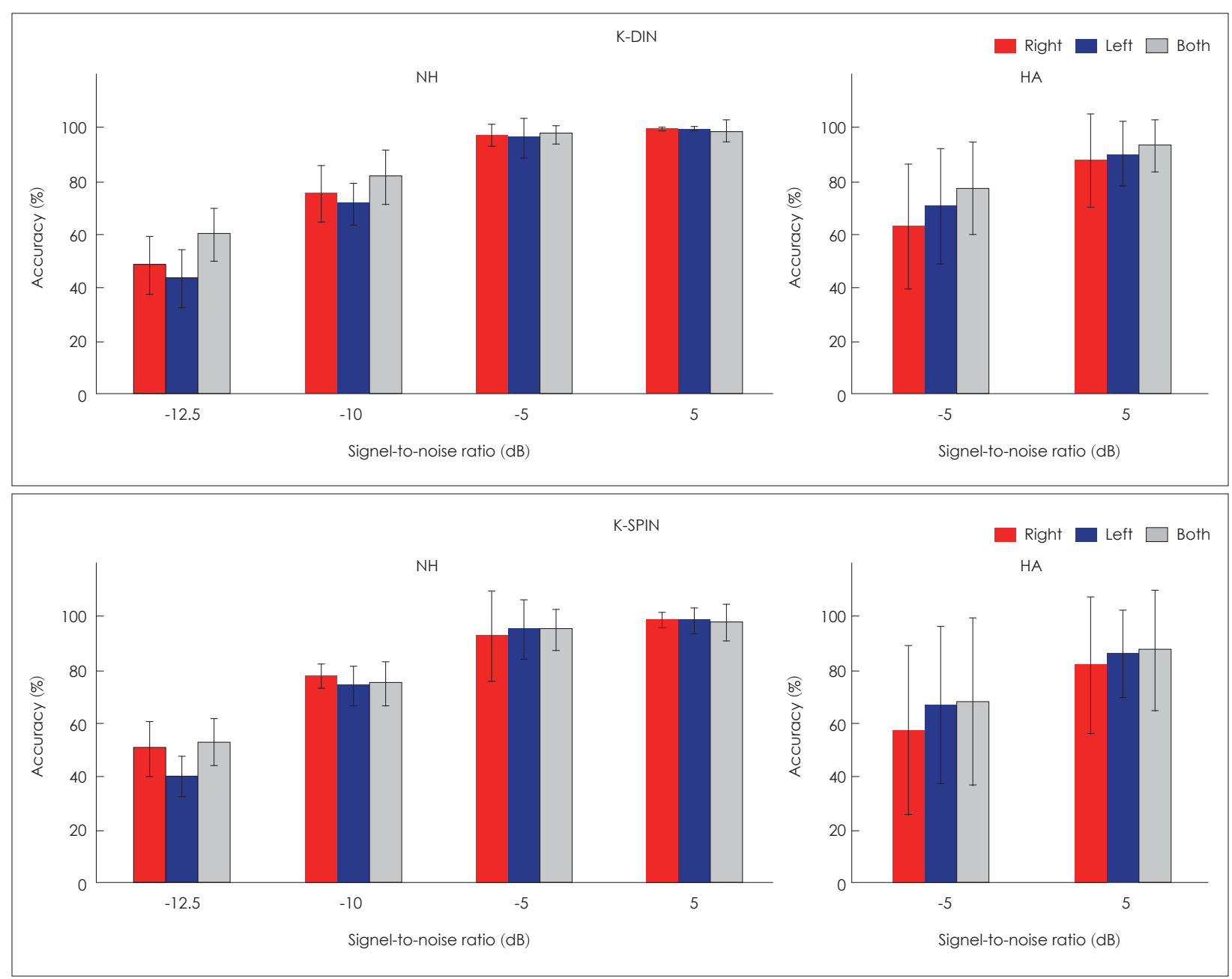

Fig. 1. Averaged speech perception accuracy for $\mathrm{NH}$ listeners and HA users as a function of signal-to-noise ratio. Upper and lower panels show results from the K-DIN and the K-SPIN tests, respectively. Error bars represent standard deviations around the mean. NH, normal hearing; HA, hearing-aid; K-DIN, Korean digits-in-noise; K-SPIN, Korean speech perception-in-noise. 
DIN is equivalent to the K-SPIN in terms of test performance and efficiency. Fig. 2 shows results by scatterplots of individual accuracy scores between K-DIN test and K-SPIN tests. In the $\mathrm{NH}$ group (Fig. 2A), the results show strong positive rela-
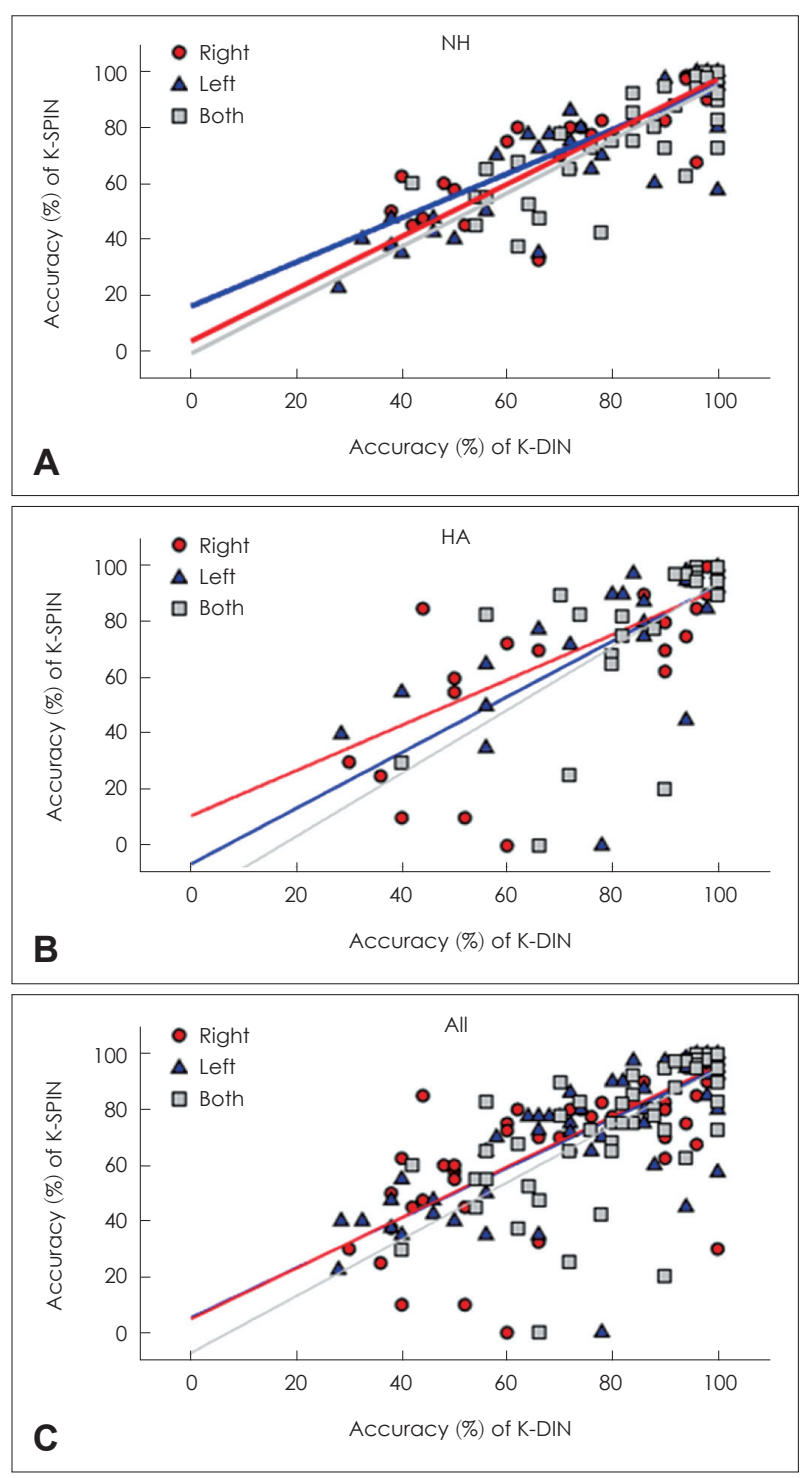

Fig. 2. Correlation between K-DIN and K-SPIN tests in NH listeners (A), HA users (B), and all subjects (C). K-DIN, Korean digits-innoise; K-SPIN, Korean speech perception-in-noise; $\mathrm{NH}$, normal hearing. tionships regardless of the hearing conditions (left: $\mathrm{r}=0.903$, $p<0.001$; right: $\mathrm{r}=0.800, p<0.001$; bilateral: $\mathrm{r}=0.827, p<0.001$ ). In the HA group (Fig. 2B), two tests are in moderate to strong positive relationships (left: $\mathrm{r}=0.627, p=0.001$; right: $\mathrm{r}=0.771$, $p<0.001$; bilateral: $\mathrm{r}=0.620, p=0.001)$. Lastly, in all subjects (Fig. 2C), the results show strong positive relationships between the K-DIN and K-SPIN tests, regardless of the hearing conditions (left: $\mathrm{r}=0.814, p<0.001$; right: $\mathrm{r}=0.788, p<0.001$; bilateral: $\mathrm{r}=0.727, p<0.001)$.

Pearson's correlation coefficient (r) and significance $(p)$ values between K-DIN and K-SPIN in each group and hearing conditions are summarized in Table 2. Overall, regardless of hearing aid usage and hearing conditions, the K-DIN has a significantly positive correlation with the K-SPIN ( $r>$ $0.727 ; p \leq 0.001$ for all cases). The positive correlation between two tests was slightly higher in the NH group compared to the HA group.

\section{Discussion}

To establish standardized diagnostic speech recognition tests across international languages, the International Collegium of Rehabilitative Audiology (ICRA) recommends the procedures and design principles for constructing, recording, optimizing, evaluating, and validating the test need to be closely matched as much as possible in each language. The ICRA recommendations are based on the comparison between the performance of the digits-in-noise test and the matrix sentence test [25]. The matrix sentence test consists of matrix words for each grammatical position (a total 50 words: 10 names, 10 verbs, 10 numerals, 10 adjectives, 10 objects). Since the combination of words results in a grammatically correct sentence, it is possible to increase the likelihood of memorizing and implementing the words in context, and makes a construction procedure difficult to validate [25]. In addition, words need to be carefully selected to reduce non-acoustic influences and must be as familiar as possible to the broadest range of the public [26].

Regardless, these considerations of constructing matrix sentence tests are not a concern for the digits-in-noise test. The

Table 2. Two-tailed Pearson's correlation coefficient $(r)$ and significance $(p)$ between K-DIN and K-SPIN tests in each group and listening conditions

\begin{tabular}{|c|c|c|c|c|c|c|}
\hline \multirow{2}{*}{ Group } & \multicolumn{2}{|c|}{ Left } & \multicolumn{2}{|c|}{ Right } & \multicolumn{2}{|c|}{ Bilateral } \\
\hline & $r$ & $p$ & $r$ & $p$ & $r$ & $p$ \\
\hline $\mathrm{NH}(\mathrm{n}=15)$ & 0.903 & $<0.001$ & 0.800 & $<0.001$ & 0.827 & $<0.001$ \\
\hline $\mathrm{HA}(\mathrm{n}=12)$ & 0.627 & 0.001 & 0.771 & $<0.001$ & 0.620 & 0.001 \\
\hline All $(n=27)$ & 0.814 & $<0.001$ & 0.788 & $<0.001$ & 0.727 & $<0.001$ \\
\hline
\end{tabular}

K-DIN, Korean digits-in-noise; K-SPIN, Korean speech perception-in-noise; NH, normal hearing; HA, hearing-aid 
ICRA recommendations of word selection, recording, synthesis, and general construction for the digits-in-noise test is relatively simple. In general, digits 0 to 9 are highly familiar even to children before school age. That is, no concern for semantic neutrality, language specific phoneme distribution or naturalness of sound of resynthesized sentence is needed. Most recently, the Canadian version of the digit triplets test was developed in both English and French [27]. Female and male versions of each English and French digit triplets test were recorded. The mean adaptive speech recognition threshold (SRT) of four language-talker versions varied by only $0.6 \mathrm{~dB}$ and the standard deviation by $0.1 \mathrm{~dB}$ despite spectral variation across two talkers and two languages. This indicates that the digit is preferable for multilingual speech tests and has a big advantage in multicultural countries.

In Korea, monosyllabic and bi-syllabic words are mainly used to estimate a subject's word recognition score and speech recognition thresholds, respectively in quiet environment. While considering daily and noisy listening condition, the KSPIN test was developed considering the characteristics of Korean sounds [6] based on an English version of the SPIN [7] at a fixed SNR. The K-HINT and the Korean matrix sentence test has also been developed by using adaptive noise level [9]. However, in actual clinical practice, these representative speech tests are not easy to perform routinely due to delivery being time consuming. As a result, both K-SPIN and K-HINT are more frequently used as auditory training tools rather than as a screening test for hearing impairment in the clinic [28]. In sum, the K-DIN as a simple speech-in-noise test will be highly useful in clinical practice in that it enables more realistic diagnosis under noise and secures time efficiency.

The Korean language is excellent as material for digits-innoise testing because of its syllabic construction. For the digit triplets test, the number of syllables for the digits must be considered to avoid allowing listeners to easily distinguish special syllable digits. In Dutch and German digit triplets tests, bisyllabic digits were omitted considering this problem [12, 16]. In Canadian version of the digit triplets test, digit " 0 " and "7" were excluded in English, and " 0 " and "4" in French [27]. However, in Korean, all syllables 0 to 9 have monosyllables so that every ten syllables can be synthesized into triplets. Further, The K-DIN does not need examiners to perform the test or grade the responses. Intuitive operation via touch screen display gives the K-DIN wide availability even for young children or those with cognitive impairments. This makes the K-DIN simple and easy to perform.

The K-DIN had been previously developed and tested by Han, et al. [22]. Their results demonstrated test-retest and training effects of the Korean digit triplet materials through various mobile devices. However, their study only focused on NH listeners, so there is a potential limitation in showing reliability of the K-DIN for hearing impaired listeners. To our knowledge, the current study is the first study to systematically investigate the correlation between the K-DIN and previously validated speech perception-in-noise testing (e.g., the K-SPIN) with both $\mathrm{NH}$ and hearing impaired listeners. Our results show a strong correlation between the two tests. Furthermore, K-DIN takes much less time compared to the $\mathrm{K}-\mathrm{SPIN}$. It can be considered that a K-DIN test can be used as a screening test. The average testing time of the K-DIN was 5 minutes, whereas K-SPIN's 30 minutes. Participants' qualitative reports also showed that the K-DIN was much easier than the K-SPIN in terms of task performance. In this aspect, the $\mathrm{K}$-DIN can be strongly considered to be used in clinic as an effective self-screening speech-in-noise test.

Digits-in-noise tests are suitable when the focus is on the lower-level auditory factors contributing to speech recognition in noise, such as during hearing aid and cochlear implant fitting [13], or the early detection and screening of hearing loss [16]. As an example, our representative HA subject (HA7), who was the only subject with severe hearing loss in our study, had poor K-SPIN performance ( $0 \%$ and $20 \%$ in -5 and $+5 \mathrm{~dB}$ SNRs, respectively). For K-DIN test, this subject scored $60 \%$ and $90 \%$ in -5 and $+5 \mathrm{~dB}$ SNRs. For this severe hearing loss subject, K-DIN can be a suitable hearing screening tool to allow frequent hearing evaluations and help with hearing aid fittings. Future work will need to involve participants with more various degrees and types of hearing loss to examine the validity of the K-DIN test.

In conclusion, digits-in-noise tests are relatively less influenced by linguistic factors, learning effects, and personal factors such as education and cognition level then the more widely used Korean sentence tests. As the first validation of the KDIN, this study shows that the K-DIN is significantly correlated with the K-SPIN for both normal-hearing and hearing-impaired listeners. These findings suggest that the K-DIN can be used as a simple and time-efficient hearing-in-noise test in audiology clinics in Korea.

\section{Acknowledgments}

This study was supported by Research Fund of Seoul St. Mary's Hospital, The Catholic University of Korea and a grant of the E.N.T. Fund of the Catholic University of Korea made in the program year of 2020. This work was also supported by the National Research Foundation of Korea(NRF) grant funded by the Korea government (MSIT) (2019R1F1A1053060).

\section{Conflicts of interest}

The authors have no financial conflicts of interest. 


\section{Author Contributions}

Conceptualization: Jae-Hyun Seo, Woojae Han, Yonghee Oh.

Data curation: Myoung Eun Sohn, Sungwha You. Formal analysis: Jae-Hyun Seo, Yonghee Oh. Funding acquisition: Jae-Hyun Seo. Investigation: Subin Kim. Methodology: Jae-Hyun Seo, Woojae Han, Yonghee Oh. Software: Yonghee Oh. Validation: Jae-Hyun Seo, Yonghee Oh. Visualization: Subin Kim. Writing — original draft: Subin Kim. Writing — review \& editing: Woojae Han, Jae-Hyun Seo, Yonghee Oh. Approval of final manuscript: all authors.

\section{ORCID iDs}

\section{Subin Kim}

Sungwha You

Myoung Eun Sohn

Woojae Han

Jae-Hyun Seo

Yonghee $\mathrm{Oh}$ https://orcid.org/0000-0003-3812-2938

https://orcid.org/0000-0003-2458-4509

https://orcid.org/0000-0001-7042-4915

https://orcid.org/0000-0003-1623-9676

https://orcid.org/0000-0002-8443-8581

https://orcid.org/0000-0002-1083-7774

\section{REFERENCES}

1) George EL, Zekveld AA, Kramer SE, Goverts ST, Festen JM, Houtgast $\mathrm{T}$. Auditory and nonauditory factors affecting speech reception in noise by older listeners. J Acoust Soc Am 2007;121:2362-75.

2) Theunissen M, Swanepoel de W, Hanekom J. Sentence recognition in noise: variables in compilation and interpretation of tests. Int $\mathrm{J}$ Audiol 2009;48:743-57.

3) Nilsson M, Soli SD, Sullivan JA. Development of the hearing in noise test for the measurement of speech reception thresholds in quiet and in noise. J Acoust Soc Am 1994;95:1085-99.

4) Killion MC, Niquette PA, Gudmundsen GI, Revit LJ, Banerjee S. Development of a quick speech-in-noise test for measuring signal-tonoise ratio loss in normal-hearing and hearing-impaired listeners. J Acoust Soc Am 2004;116:2395-405.

5) Kollmeier B, Warzybok A, Hochmuth S, Zokoll MA, Uslar V, Brand $\mathrm{T}$, et al. The multilingual matrix test: principles, applications, and comparison across languages: a review. Int J Audiol 2015;54 Suppl 2:3-16.

6) An LJ, Kim JS, Pae SY. The study on developing a test of speech perception in noise. Korean J Audiol 2002;6:118-27.

7) Kalikow DN, Stevens KN, Elliott LL. Development of a test of speech intelligibility in noise using sentence materials with controlled word predictability. J Acoust Soc Am 1977;61:1337-51.

8) Moon SK, Mun HA, Jung HK, Soli SD, Lee JH, Park K. Development of sentences for Korean hearing in noise test (KHINT). Korean J Otolaryngol-Head Neck Surg 2005;48:724-8.

9) Kim KH, Lee JH. Evaluation of the Korean matrix sentence test: verification of the list equivalence and the effect of word position. Audiol Speech Res 2018;14:100-7.

10) Kaandorp MW, Smits C, Merkus P, Goverts ST, Festen JM. Assessing speech recognition abilities with digits in noise in cochlear implant and hearing aid users. Int J Audiol 2015;54:48-57.

11) Vlaming MS, MacKinnon RC, Jansen M, Moore DR. Automated screening for high-frequency hearing loss. Ear Hear 2014;35:667-79.

12) Smits $C$, Kapteyn TS, Houtgast T. Development and validation of an automatic speech-in-noise screening test by telephone. Int J Audiol 2004;43:15-28.

13) Smits $C$, Theo Goverts S, Festen JM. The digits-in-noise test: assessing auditory speech recognition abilities in noise. J Acoust Soc Am 2013;133:1693-706.

14) Hall JS. The development of a new English sentence in noise test and an English number recognition test [dissertation]. Southampton: University of Southampton;2006.

15) Watson CS, Kidd GR, Miller JD, Smits C, Humes LE. Telephone screening tests for functionally impaired hearing: current use in seven countries and development of a US version. J Am Acad Audiol 2012;23:757-67.

16) Zokoll MA, Wagener KC, Brand T, Buschermöhle M, Kollmeier B. Internationally comparable screening tests for listening in noise in several European languages: the German digit triplet test as an optimization prototype. Int J Audiol 2012;51:697-707.

17) Jansen S, Luts H, Dejonckere P, van Wieringen A, Wouters J. Exploring the sensitivity of speech-in-noise tests for noise-induced hearing loss. Int J Audiol 2014;53:199-205.

18) Zhang H, Wang S, Wang L, Chen J, Yue P, Guo L, et al. Digital recording and equivalent evaluation of Mandarin speech test materials. Journal of Clinical Otorhinolaryngology 2006;20:1011-5.

19) Ozimek E, Kutzner D, Sęk A, Wicher A. Development and evaluation of Polish digit triplet test for auditory screening. Speech Commun 2009;51:307-16.

20) Warzybok A, Zokoll MA, Kollmeier B. Development and evaluation of the Russian digit triplet test. Acta Acust united Ac 2016;102:714-24.

21) Pérez-González P, Gorospe JM, Lopez-Poveda EA. A Castilian Spanish digit triplet identification test for assessing speech intelligibility in quiet and in noise. Revista de acústica 2013;44:13-24.

22) Han JH, Yi DW, Lee J, Chang WD, Lee HJ. Development of a smartphone-based digits-in-noise test in Korean: a hearing screening tool for speech perception in noise. J Korean Med Sci 2020;35:e163.

23) Keidser G, Dillon H, Flax M, Ching T, Brewer S. The NAL-NL2 prescription procedure. Audiol Res 2011;1:e24.

24) Kim TH, Jhoo JH, Park JH, Kim JL, Ryu SH, Moon SW, et al. Korean version of mini mental status examination for dementia screening and its' short form. Psychiatry Investig 2010;7:102-8.

25) Akeroyd MA, Arlinger S, Bentler RA, Boothroyd A, Dillier N, Dreschler WA, et al. International collegium of rehabilitative audiology (ICRA) recommendations for the construction of multilingual speech tests. ICRA working group on multilingual speech tests. Int J Audiol 2015;54 Suppl 2:17-22.

26) Neumann K, Baumeister N, Baumann U, Sick U, Euler HA, Weissgerber T. Speech audiometry in quiet with the Oldenburg sentence test for children. Int J Audiol 2012;51:157-63.

27) Giguère C, Lagacé J, Ellaham NN, Pichora-Fuller MK, Goy H, Bégin C, et al. Development of the Canadian digit triplet test in English and French. J Acoust Soc Am 2020;147:EL252.

28) Yu J, Chun H, Song CG, Han W. Case study of speech perception enhancement in hearing-impaired adult by auditory training program of mobile device. Audiol 2014;10:158-68. 